CORRECTION

\title{
Correction: Risk factors for H5 avian influenza virus prevalence on urban live bird markets in Jakarta, Indonesia-Evaluation of long-term environmental surveillance data
}

\section{The PLOS ONE Staff}

In Table 1, the headings Mean (SD) H5 negative and Mean (SD) H5 positive do not appear in the correct columns. The publisher apologizes for the error. Please see the correct Table 1 here.

f open Access

Citation: The PLOS ONE Staff (2019) Correction: Risk factors for $\mathrm{H} 5$ avian influenza virus prevalence on urban live bird markets in Jakarta, IndonesiaEvaluation of long-term environmental surveillance data. PLOS ONE 14(8): e0221611. https://doi.org/ 10.1371/journal.pone.0221611

Published: August 20, 2019

Copyright: ๑ 2019 The PLOS ONE Staff. This is an open access article distributed under the terms of the Creative Commons Attribution License, which permits unrestricted use, distribution, and reproduction in any medium, provided the original author and source are credited. 
Table 1. Final multivariable model results for risk factors associated with HPAI H5 virus prevalence at live bird markets in the Greater Jakarta Region, Indonesia, between March 2009 and July 2014.

\begin{tabular}{|c|c|c|c|c|c|c|c|c|c|}
\hline \multirow[t]{2}{*}{ RISK FACTORS } & \multirow[t]{2}{*}{ Level } & \multicolumn{2}{|c|}{ N Observations (percent) } & \multicolumn{3}{|c|}{ Univariate analysis } & \multicolumn{3}{|c|}{ Multivariable analysis } \\
\hline & & H5 negative & H5 positive & OR & P-value & \begin{tabular}{|c|} 
Wald test \\
P-value
\end{tabular} & OR & P-value & $\begin{array}{l}\text { Wald test } \\
\text { P-value }\end{array}$ \\
\hline \multicolumn{10}{|l|}{ MARKET CHARACTERISTICS } \\
\hline \multirow[t]{8}{*}{ Market layout ${ }^{1}$} & A & $934(64.3 \%)$ & $518(35.7 \%)$ & 1 & & $<0.001$ & 1 & & $<0.001$ \\
\hline & B & $537(58.3 \%)$ & $384(35.7 \%)$ & $\begin{array}{c}1.3(0.8 \\
2.3)\end{array}$ & 0.322 & & $\begin{array}{c}1.1(0.7 \\
1.6)\end{array}$ & 0.786 & \\
\hline & $\mathrm{C}$ & $169(56.1 \%)$ & $132(43.9 \%)$ & $\begin{array}{c}1.3(0.8 \\
2.0)\end{array}$ & 0.268 & & $\begin{array}{c}1.5(1.0 \\
2.2)\end{array}$ & 0.060 & \\
\hline & $\mathrm{D}$ & $33(34.4 \%)$ & $63(65.6 \%)$ & $\begin{array}{c}3.3(2.3 \\
4.9)\end{array}$ & $<0.001$ & & $\begin{array}{c}1.4(0.8 \\
2.3)\end{array}$ & 0.229 & \\
\hline & $\mathrm{E}$ & $58(57.4 \%)$ & $43(42.6 \%)$ & $\begin{array}{c}1.3(0.7 \\
2.4)\end{array}$ & 0.358 & & $\begin{array}{c}1.1(0.5 \\
2.2)\end{array}$ & 0.829 & \\
\hline & $\mathrm{F}$ & $120(79.5 \%)$ & $31(20.5 \%)$ & $\begin{array}{c}0.3(0.1 \\
0.9)\end{array}$ & 0.03 & & $\begin{array}{c}0.4(0.2 \\
1.1)\end{array}$ & 0.071 & \\
\hline & SALE only 1 & $159(56.0 \%)$ & $125(44.0 \%)$ & $\begin{array}{c}1.4(0.7 \\
2.8)\end{array}$ & 0.377 & & $\begin{array}{c}1.3(0.7 \\
2.1)\end{array}$ & 0.417 & \\
\hline & SALE only 2 & $254(93.0 \%)$ & $19(7.0 \%)$ & $\begin{array}{c}0.1(0.0 \\
0.3)\end{array}$ & $<0.001$ & & $\begin{array}{c}0.2(0.1 \\
0.5)\end{array}$ & 0.002 & \\
\hline \multirow{5}{*}{$\begin{array}{l}\text { Most dominant poultry species on the } \\
\text { market }\end{array}$} & Broilers & $1,592(66.4 \%)$ & $807(33.6 \%)$ & 1 & & $<0.001$ & 1 & & $<0.001$ \\
\hline & Layers & $92(66.2 \%)$ & $47(33.8 \%)$ & $\begin{array}{c}1.0(0.7 \\
1.5)\end{array}$ & 0.849 & & $\begin{array}{c}0.7(0.5 \\
1.0)\end{array}$ & 0.054 & \\
\hline & $\begin{array}{l}\text { Kampung } \\
\text { Chickens }\end{array}$ & $435(56.3 \%)$ & $337(43.7 \%)$ & $\begin{array}{c}1.5(0.8 \\
2.9)\end{array}$ & 0.243 & & $\begin{array}{c}1.3(0.8 \\
2.2)\end{array}$ & 0.355 & \\
\hline & Ducks & $120(54.5 \%)$ & $100(45.5 \%)$ & $\begin{array}{c}1.6(0.8 \\
3.0)\end{array}$ & 0.149 & & $\begin{array}{c}0.9(0.5 \\
1.9)\end{array}$ & 0.868 & \\
\hline & Parent stock & $25(51.0 \%)$ & $24(49.0 \%)$ & $\begin{array}{c}2.0(1.5 \\
2.7)\end{array}$ & $<0.001$ & & $\begin{array}{c}5.7(3.6 \\
9.2)\end{array}$ & $<0.001$ & \\
\hline \multicolumn{10}{|l|}{ POULTRY MANAGEMENT ON MARKET } \\
\hline \multirow[t]{2}{*}{ Display tables made from wood } & No & $1,032(58.3 \%)$ & $739(41.7 \%)$ & 1 & & & & & \\
\hline & Yes & $1,232(68.1 \%)$ & $576(31.9 \%)$ & $\begin{array}{c}0.6(9.3 \\
0.9)\end{array}$ & 0.018 & & $\begin{array}{c}0.7(0.5 \\
1.0)\end{array}$ & 0.047 & \\
\hline \multicolumn{10}{|l|}{ SAMPLING CHARACTERISTICS } \\
\hline \multirow[t]{2}{*}{ Samples obtained from at least one duck } & No & $2,083(65.8 \%)$ & $1,083(34.2 \%)$ & 1 & & & & & \\
\hline & Yes & $181(43.8 \%)$ & $232(56.2 \%)$ & $\begin{array}{c}1.5(1.1 \\
2.1)\end{array}$ & 0.019 & & $\begin{array}{c}1.6(1.1 \\
2.3)\end{array}$ & 0.009 & \\
\hline \multirow{2}{*}{\multicolumn{2}{|c|}{ ENVIRONMENTAL FACTORS }} & \multicolumn{2}{|c|}{ Mean (SD) } & & & & & & \\
\hline & & H5 negative & H5 positive & & & & & & \\
\hline \multicolumn{2}{|c|}{$\begin{array}{l}\text { Human population density in the district ( } \mathrm{N} \text { people/square } \\
\text { kilometre) }\end{array}$} & $\begin{array}{c}9,018.3 \\
(5,023.0)\end{array}$ & $\begin{array}{l}11,958.9 \\
(4,379.6)\end{array}$ & $\begin{array}{c}2.2(1.8 \\
2.7)^{2}\end{array}$ & $<0.001$ & & $\begin{array}{c}1.6(1.3 \\
1.9)^{2}\end{array}$ & $<0.001$ & \\
\hline \multicolumn{2}{|c|}{$\begin{array}{l}\text { Average distance between market and origin of poultry sold } \\
\text { at the market (kilometre) }\end{array}$} & $16.8(35.7)$ & $35.9(47.8)$ & $\begin{array}{c}1.6(1.2, \\
2.0)^{2}\end{array}$ & $<0.001$ & & $\begin{array}{c}1.3(1.1 \\
1.6)^{2}\end{array}$ & 0.011 & \\
\hline \multicolumn{2}{|l|}{ Total rainfall per month (mm) } & $94.2(87.1)$ & $113.3(94.4)$ & $\begin{array}{c}1.2(1.2, \\
1.3)^{2}\end{array}$ & $<0.001$ & & $\begin{array}{l}1.3(1.2, \\
1.4)^{2}\end{array}$ & $<0.001$ & \\
\hline
\end{tabular}

${ }^{1}$ Live Bird Market layouts: A = Slaughter, sale of live birds and carcass sales in same area, B = Slaughter and live bird sales conducted outside and carcass sales inside, $\mathrm{C}=$ Slaughter, sale of live birds and carcass sales in same areas, but separated by individual partitions, $\mathrm{D}=$ Slaughter and live bird sales in same areas inside, and separated from carcass sales by screens, $\mathrm{E}=$ Slaughter, live bird and carcass sales inside in separate areas, but no protective screens, $\mathrm{F}=$ Live birds sale outside and slaughter and carcass sales inside and in separate areas, Sale 1 only $=$ No slaughtering at the market, but slaughter facility in vicinity of the market, Sale 2 only $=$ No slaughtering at the market, and slaughter facility far away from the market

${ }^{2}$ Odds ratios and confidence intervals are displayed for standardized values

https://doi.org/10.1371/journal.pone.0221611.t001 


\section{PLOS $\mid$ ONE}

\section{Reference}

1. Henning J, Hesterberg UW, Zenal F, Schoonman L, Brum E, McGrane J (2019) Risk factors for H5 avian influenza virus prevalence on urban live bird markets in Jakarta, Indonesia-Evaluation of longterm environmental surveillance data. PLoS ONE 14(5): e0216984. https://doi.org/10.1371/journal. pone.0216984 PMID: 31125350 